\section{Wave-Guide Systems with Negative Phase Velocities}

Is all smooth wave-guides and many loaded wave-guides the phase velocity has the same sign as the energy velocity. Some systems having complex forms of loading have in the past been attributed with zero or even negative group-velocities, based on apparently anomalous dispersion curves. Such phenomena have been clouded by doubts about the conception of group velocity and its relationship to energy velocity. It has recently been shown that in a periodic structure with negligible attenuation, energy and group velocities are identical ${ }^{1}$. If a system is analysed in terms of the positive solution for phase velocity, a zero group-velocity indicates a form of resonance with zero net power flow, and a negative group-velocity indicates a negative net energy velocity. Since in any experiment the net energy velocity is taken to be positive, systems can be devised in which the phase-velocity is negative. Dispersion curves showing guide and air wave-lengths as ordinates and abscissæ must have a positive slope, and in these anomalous systems this requires a negative sign to be attributed to the measured guide wave-lengths. When interaction with charged particles is required, it is of supreme importance to determine the correct direction of the phase-velocity.

In such cases as the helix and the folded-strip transmission line, in which a wave with positive phase and energy velocities flows along the folded line, the fundamental wave (defined as that with longest wave-length), as observed by a charged particle travelling parallel to the axis of net flow of power, can have a genuinely negative phase-velocity. If a standing wave in any system is observed at discrete points by a squarelaw detector, the correctly drawn wavelength curves together with those obtained by adding $\pm 2 n \pi$ phase change between successive points will show the correct phase velocities for a fundamental and its associated space harmonics, all with the same group-velocity equal to the totalenergy velocity. Wave-length curves can, however, be drawn through such points with t: $(2 n+1) \pi$ between successive points, and erroneous phaseand energy-velocities are then obtained.

There are, however, structures in which there can be no such confusion of space harmonics or wrongly drawn standing-wave curves, where the fundamental wave (defined as the mode which carries all the power when the periodic length is infinitely small) has a negative phase velocity.

A rectangular wave-guide which is loaded on one broad face with a reactive sheet propagates a longitudinal section wave $\left(E_{11} H_{11}\right)$ with either positive or negative phase-velocity, depending upon whether the broad dimension is greater or less than $\lambda / 2$ ( $\lambda$ being the wave-length in air). The way in which the square of the propagation constant $(2 \pi /$ guide wave-length) changes with the reactance of the sheet is shown in the diagram. To obtain a 'slow' wave with negative phase-velocity the reactive sheet must be capacitative, as compared with inductive for a positive phase-velocity.
The abnormal behaviour below $H_{01}$ cut-off has been confirmed by two experimental arrangements : (1) a corrugated rectangular wave-guide in which the common broad dimension was less than $\lambda / 2$ and the corrugations were made above $H_{01}$ cut-off by loading with a dielectric material; (2) a rectangular waveguide of broad dimension less than $\lambda / 2$ loaded between the centres of its narrow faces by a slotted plate, the effective width of the slots being raised above $\lambda / 2$ by thickening the plate along its centre line. In the latter system, the stored energy and power losses for a given electric field can be smaller than in the normal corrugated wave-guide, especially at very low phase-velocities, such as are required. for travelling-wave tubes and for heavy-particle accelerators. 